\title{
SEMI-CONTINUITY FOR DERIVED CATEGORIES
}

\author{
YURIY DROZD
}

\begin{abstract}
We prove that the number of parameters defining a complex of projective modules over an algebra is upper semi-continuous in families of algebras. Supposing that every algebra is either derived tame or derived wild, we get that a degeneration of a derived wild algebra is also derived wild. The proof follows the pattern of the paper and rests upon universal families with projective bases. We also explain why the so-called counter-example of Brüstle [2] is in fact not a counter-example.
\end{abstract}

In the representation theory of finite dimensional algebras it is usual to distinguish three types of algebras: representation finite, tame and wild (cf. [6, 国). A useful tool in establishing representation type is provided by deformation theory. It is based on upper semi-continuity of parameter number $\operatorname{par}(n, \mathbf{A}(x))$ defining an $n$-dimensional module over an algebra $\mathbf{A}(x)$, when $\mathbf{A}(x)$ is an algebraic family of algebras (see [7, 5]). During last years analogous investigation of derived categories has been started, especially derived tame and wild algebras have been considered. In this article we define parameter numbers of complexes and prove that they are also upper semi-continuous in families of algebras. We follow the technique elaborated in [5] and going back to a paper of H. Knörrer [12]. Especially, our proof depends on the construction of (almost) universal families of complexes with projective bases. As a corollary, we prove that if an algebra, which is not derived tame, degenerates to another algebra, the latter is also not derived tame (note that most people working on the subject believe that 'not tame' means 'wild' in this situation too). Recently Th. Brüstle [2] has announced a counter-example to the last assertion. We explain why it is actually not a counter-example.

\section{Categories $\mathcal{K}^{n}(\mathbf{A})$}

Let $\mathbf{A}$ be a ring. We denote by Mod-A (mod-A) the category of right $\mathbf{A}$-modules (respectively, of finitely generated $\mathbf{A}$-modules). We define the category $\mathcal{K}^{n}(\mathbf{A})$ as follows.

1. Its objects are finite complexes of projective A-modules

$$
P_{\bullet}: \quad P_{n} \stackrel{d_{n}}{\longrightarrow} P_{n-1} \stackrel{d_{n-1}}{\longrightarrow} \ldots \longrightarrow P_{m}
$$

$(m \leq n)$. We set $P_{k}=0$ for $k<m$.

2. Morphisms in $\mathcal{K}^{n}(\mathbf{A})$ are homomorphisms of complexes modulo quasi-homotopy. Namely, two homomorphisms $\phi=\left(\phi_{k}\right)$ and $\psi=\left(\psi_{k}\right)$ from $P_{\bullet}$ to $P_{\bullet}^{\prime}$

2000 Mathematics Subject Classification. 16G10; 16E05.

This work was inspired by my visit to the University of California at Santa Barbara and accomplished during my stay at the University of Kaiserslautern. I thank the host Universities and the supporting foundations, CRDF (Award UM2-2094) and DFG (Schwerpunkt "Globale Methoden in der komplexen Geometrie") for this opportunity. I am also grateful to Birge HuisgenZimmermann and Igor Burban for useful discussions. 
are called quasi-homotopic if there are homomorphisms of modules $s_{k}: P_{k} \rightarrow$ $P_{k+1}^{\prime}$ such that $\psi_{k}=\phi_{k}+s_{k-1} d_{k}+d_{k+1}^{\prime} s_{k}$ for all $k<n$. We write $\phi \stackrel{n}{\sim} \psi$ in this case and call $s=\left(s_{k}\right)$ a quasi-homotopy from $\phi$ to $\psi$.

Note that the last homomorphisms $\phi_{n}, \psi_{n}$ do not influence quasi-homotopy at all.

There is a natural functor $\mathcal{I}_{n}: \mathcal{K}^{n}(\mathbf{A}) \rightarrow \mathcal{K}^{n+1}(\mathbf{A})$. Namely, if $P_{\bullet}$ is a complex from $\mathcal{K}^{n}$, choose an epimorphism $d_{n+1}: P_{n+1} \rightarrow P_{n}$ with $\operatorname{Im} d_{n+1}=\operatorname{Ker} d_{n}$ and define $\mathcal{I}_{n} P_{\bullet}$ as the complex

$$
P_{n+1} \stackrel{d_{n+1}}{\longrightarrow} P_{n} \stackrel{d_{n}}{\longrightarrow} \ldots \longrightarrow P_{m} .
$$

If $\phi$ is a homomorphism $P_{\bullet} \rightarrow P_{\bullet}^{\prime}$, we can lift it to a homomorphism $\mathcal{I}_{n} \phi: \mathcal{I}_{n} P \bullet \bullet$ $\mathcal{I}_{n} P_{\bullet}^{\prime}$ in a usual way. Moreover, if $\phi \stackrel{n}{\sim} \psi$ and $s=\left(s_{n}\right)$ is a corresponding quasihomotopy, one easily sees that $d_{n}^{\prime}\left(\phi_{n}-\psi_{n}-s_{n-1} d_{n}\right)=0$, hence there is a mapping $s_{n}: P_{n} \rightarrow P_{n+1}^{\prime}$ such that $\phi_{n}-\psi_{n}-s_{n-1} d_{n}=d_{n+1}^{\prime} s_{n}$, so we get a quasi-homotopy $\mathcal{I}_{n} \phi \stackrel{n+1}{\sim} \mathcal{I}_{n} \psi$. Therefore the functor $\mathcal{I}_{n}$ is well defined. It gives rise to the direct limit $\mathcal{K}^{\omega}(\mathbf{A})=\lim _{\longrightarrow} \mathcal{K}^{n}(\mathbf{A})$.

Proposition 1.1. The category $\mathcal{K}^{\omega}(\mathbf{A})$ is equivalent to the bounded derived category $D^{b}$ (Mod-A). If $\mathbf{A}$ is noetherian, the bounded derived category $D^{b}(\bmod -\mathbf{A})$ is equivalent to the full subcategory $\mathcal{K}_{f}^{\omega}(\mathbf{A})=\lim _{n} \mathcal{K}_{f}^{n}(\mathbf{A})$ of $\mathcal{K}^{\omega}(\mathbf{A})$, where $\mathcal{K}_{f}^{n}(\mathbf{A})$ is the full subcategory of $\mathcal{K}^{n}(\mathbf{A})$ consisting of complexes of finitely generated modules.

(This result traces back to the paper [11.)

Proof. Consider the functor $\mathcal{J}_{n}: \mathcal{K}^{n}(\mathbf{A}) \rightarrow D^{b}$ (Mod-A), which maps a complex $P$ • to the complex

$$
\text { Ker } d_{n} \longrightarrow P_{n} \stackrel{d_{n}}{\longrightarrow} P_{n-1} \stackrel{d_{n-1}}{\longrightarrow} \ldots \longrightarrow P_{m} .
$$

One can check that $\mathcal{J}_{n+1} \mathcal{I}_{n} \simeq \mathcal{J}_{n}$, so we get the limit functor $\mathcal{J}=\underline{\lim }_{n} \mathcal{J}_{n}$ : $\mathcal{K}^{\omega}(\mathbf{A}) \rightarrow D^{b}$ (Mod-A). On the other hand, let $C$ • be a complex from $D^{b}(\operatorname{Mod}-\mathbf{A})$ such that $C_{k}=0$ for $k \geq n$. Consider its projective resolution $P_{\bullet}$; it is exact at all $P_{k}$ with $k \geq n$. Let $P_{\bullet}^{(n)} \in \mathcal{K}^{n}(\mathbf{A})$ be the complex that coincide with $P_{\bullet}$ for $k \leq n$. Then $C_{\bullet} \simeq \mathcal{J}_{n} P_{\bullet}^{(n)}$ in $D^{b}(\operatorname{Mod}-\mathbf{A})$. Moreover, $\mathcal{I}_{n} P_{\bullet}^{(n)} \simeq P_{\bullet}^{(n+1)}$, so $C_{\bullet} \simeq \mathcal{J} P^{(n)}$ and we get a functor $D^{b}(\operatorname{Mod}-\mathbf{A}) \rightarrow \mathcal{K}^{\omega}(\mathbf{A})$ inverse to $\mathcal{J}$.

The assertion about noetherian case is obvious.

Note that there are also natural functors $\mathcal{E}_{n}: \mathcal{K}^{n+1}(\mathbf{A}) \rightarrow \mathcal{K}^{n}(\mathbf{A})$ : we just omit the term $P_{n+1}$. Hence the inverse limit $\mathcal{K}^{\infty}(\mathbf{A})=\lim _{n} \mathcal{K}^{n}(\mathbf{A})$ is defined and the following result hold.

Proposition 1.2. The category $\mathcal{K}^{\infty}(\mathbf{A})$ is equivalent to the right bounded derived category $D^{-}$(Mod-A). If $\mathbf{A}$ is noetherian, the right bounded derived category $D^{-}(\bmod -\mathbf{A})$ is equivalent to the full subcategory $\mathcal{K}_{f}^{\infty}(\mathbf{A})=\lim _{n} \mathcal{K}_{f}^{n}(\mathbf{A})$ of $\mathcal{K}^{\infty}(\mathbf{A})$.

The proof is quite analogous to that of Proposition 1.1 and we omit it.

Suppose now that the ring $\mathbf{A}$ is semiperfect [1]; set $\mathbf{R}=\operatorname{rad} \mathbf{A}$. Then any right bounded complex of finitely generated projective $\mathbf{A}$-modules is homotopic to a minimal complex $P_{\bullet}$, i.e. such that $\operatorname{Im} d_{n} \subseteq P_{n-1} \mathbf{R}$ for all $n$. Thus, considering $\mathcal{K}_{f}^{n}(\mathbf{A})$, we may confine ourselves to minimal complexes, and we shall always do so. Note that two minimal complexes are homotopic if and only if they are isomorphic. We call a minimal complex (1.1) reduced if $\operatorname{Ker} d_{n} \subseteq P_{n}$. Any complex from $\mathcal{K}_{f}^{n}(\mathbf{A})$ 
is isomorphic (as complex) to a direct sum of a reduced one and a complex having all zero components except maybe the $n$ th. The latter is a zero object of $K^{n}(\mathbf{A})$, so any complex is isomorphic in $\mathcal{K}_{f}^{n}(\mathbf{A})$ to a reduced one. Moreover, if a homomorphism $\phi: P_{\bullet} \rightarrow P_{\bullet}^{\prime}$ of reduced complexes from $\mathcal{K}_{f}^{n}(\mathbf{A})$ is quasi-homotopic to $0, \operatorname{Im} \phi_{k} \subseteq$ $P_{k}^{\prime} \mathbf{R}$ for all $k$ including $k=n$. Therefore if two reduced complexes are isomorphic in $\mathcal{K}_{f}^{n}(\mathbf{A})$, they are isomorphic as complexes.

We denote by $\mathcal{C}^{n}(\mathbf{A})$ the category of minimal complexes $P_{\bullet}$ with $P_{k}=0$ for $k>n$ and by $\mathcal{C}_{0}^{n}(\mathbf{A})$ its full subcategory of reduced complexes. Then the natural functor $\mathcal{C}^{n}(\mathbf{A}) \rightarrow \mathcal{K}_{f}^{n}(\mathbf{A})$ induces a representation equivalence of $\mathcal{C}_{0}^{n}(\mathbf{A})$ onto $\mathcal{K}_{f}^{n}(\mathbf{A})$. Here we call a functor $F: \mathcal{A} \rightarrow \mathcal{B}$ a representation equivalence, if

- $F$ is dense, i.e. every object from $\mathcal{B}$ is isomorphic to $F A$ for some object $A \in \mathcal{A}$;

- $F$ is full, i.e. all induced mappings $\mathcal{A}\left(A, A^{\prime}\right) \rightarrow \mathcal{B}\left(F A, F A^{\prime}\right)$ are surjective;

- $F$ is conservative, i.e. $F \phi$ is an isomorphism if and only if $\phi$ is an isomorphism.

An evident consequence of these conditions is

- $F A$ is indecomposable if and only if $A$ is indecomposable.

Let $A_{1}, A_{2}, \ldots, A_{t}$ be all pairwise non-isomorphic indecomposable projective A-modules (all of them are direct summands of $\mathbf{A}$ ). If $P$ is a finitely generated projective $\mathbf{A}$-module, it uniquely decomposes as $\bigoplus_{i=1}^{t} p_{i} A_{i}$. We denote by $\mathbf{r}(P)$ the vector $\left(p_{1}, p_{2}, \ldots, p_{t}\right)$ and call it the rank of $P$. For any complex (1.1) from $\mathcal{C}^{n}(\mathbf{A})$ we denote by $\mathbf{r}_{\bullet}\left(P_{\bullet}\right)$ and call the vector rank of $P_{\bullet}$ the sequence $\left(\mathbf{r}\left(P_{n}\right), \mathbf{r}\left(P_{n-1}\right), \ldots, \mathbf{r}\left(P_{m}\right)\right)$. As we have already remarked, every complex from $\mathcal{C}^{n}(\mathbf{A})$ decomposes as $P_{\bullet} \oplus\left(\bigoplus_{i=1}^{t} a_{i} A_{i}[n]\right)$, where $P_{\bullet}$ is a reduced complex and $A[n]$ denotes, as usually, the complex with a unique non-zero component, namely the $n$th one, equal $A$. Thus, from the viewpoint of classification problem, there is no essential difference between $\mathcal{C}^{n}(\mathbf{A})$ and $\mathcal{K}_{f}^{n}(\mathbf{A})$.

Given a vector $\mathbf{r}=\left(p_{1}, p_{2}, \ldots, p_{t}\right)$, we denote $\mathbf{r} A=\bigoplus_{i=1}^{t} p_{i} A_{i}$ and set $\mathbf{A}\left(\mathbf{r}, \mathbf{r}^{\prime}\right)=$ $\operatorname{Hom}_{\mathbf{A}}\left(\mathbf{r} A, \mathbf{r}^{\prime} A\right), \mathbf{R}\left(\mathbf{r}, \mathbf{r}^{\prime}\right)=\operatorname{Hom}_{\mathbf{A}}\left(\mathbf{r} A, \mathbf{r}^{\prime} A \mathbf{R}\right)$. For any sequence $\left(\mathbf{r}_{n}, \mathbf{r}_{n-1}, \ldots, \mathbf{r}_{m}\right)$ we consider the set $\mathcal{C}^{n}(\mathbf{r})$ of minimal complexes

$$
\mathbf{r}_{n} A \stackrel{d_{n}}{\longrightarrow} \mathbf{r}_{n-1} A \stackrel{d_{n-1}}{\longrightarrow} \ldots \longrightarrow \mathbf{r}_{m} A
$$

or, the same, of sequences $\left(d_{n}, d_{n-1}, \ldots, d_{m+1}\right)$ with $d_{k} \in \mathbf{R}\left(\mathbf{r}_{k}, \mathbf{r}_{k-1}\right)$ such that $d_{k} d_{k+1}=0$ for all $m<k<n$. Two sequences $\left(d_{k}\right)$ and $\left(d_{k}^{\prime}\right)$ define isomorphic complexes if and only if there are invertible mappings $\alpha_{k} \in \mathbf{A}\left(\mathbf{r}_{k}, \mathbf{r}_{k}\right)(m \leq k \leq n)$ such that $\alpha_{k-1} d_{k}=d_{k}^{\prime} \alpha_{k}$ for all $m<k \leq n$. Especially two sequences $\left(d_{n}\right)$ and $\left(\lambda_{n} d_{n}\right)$, where $\lambda_{n}$ are invertible elements from the centre of $\mathbf{A}$, always define isomorphic complexes.

If $\mathbf{A}$ is a finite dimensional algebra over a field $\mathbb{k}$, it allows us to consider complexes from $\mathcal{C}^{n}(\mathbf{A})$ of a fixed vector rank $\mathbf{r}_{\mathbf{r}}$ as ( $\mathbb{k}$-valued) points of an algebraic variety $\mathcal{C}\left(\mathbf{r}_{\bullet}\right)$, which is a subvariety of $\mathcal{H}\left(\mathbf{r}_{\bullet}\right)=\prod_{k=m+1}^{n} \mathbf{R}\left(\mathbf{r}_{k}, \mathbf{r}_{k+1}\right)$. Moreover, homothetic sequences $\left(d_{n}\right)$ and $\left(\lambda d_{n}\right)$ define isomorphic complexes, so considering the classification problem we may replace the vector space $\mathcal{H}\left(\mathbf{r}_{\bullet}\right)$ by the projective space $\mathbb{P}\left(\mathbf{r}_{\bullet}\right)=\mathbb{P}\left(\mathcal{H}\left(\mathbf{r}_{\bullet}\right)\right)$ and $\mathcal{C}\left(\mathbf{r}_{\bullet}\right)$ by its image $\mathbb{D}\left(\mathbf{r}_{\bullet}\right)$ in $\mathbb{P}\left(\mathbf{r}_{\bullet}\right)$, which is a projective variety. 


\section{FAMILIES OF COMPLEXES}

From now on let $\mathbf{A}$ be a finite dimensional algebra over an algebraically closed field $\mathbb{k}$ and $\mathbf{I} \subseteq \mathbf{R}$ be an ideal. We define an $\mathbf{I}$-family of $\mathbf{A}$-complexes over an algebraic variety $X$ as a complex of flat coherent sheafs of $\mathbf{A}_{X}$-modules

$$
\mathcal{P}_{\bullet}: \quad \mathcal{P}_{n} \stackrel{d_{n}}{\longrightarrow} \mathcal{P}_{n-1} \stackrel{d_{n-1}}{\longrightarrow} \ldots \longrightarrow \mathcal{P}_{m},
$$

where $\mathbf{A}_{X}=\mathcal{O}_{X} \otimes \mathbf{A}$, such that $\operatorname{Im} d_{k} \subseteq \mathcal{P}_{k-1} \mathbf{I}$ for all $m<k \leq n$. If $\mathbf{I}=\mathbf{R}$, we also call such a complex a family of minimal $\mathbf{A}$-complexes. Given a family $\mathcal{P}_{\bullet}$ and a point $x \in X$, we get a complex $\mathcal{P}_{\bullet}(x)$ from $\mathcal{C}^{n}(\mathbf{A})$. Since $\mathcal{P}_{n}$ are locally free over $\mathcal{O}_{X}$, the $\operatorname{ranks} \mathbf{r}\left(\mathcal{P}_{k}(x)\right)$ are locally constant. We usually suppose $X$ connected; then these ranks are constant, so we can define $\mathbf{r}\left(\mathcal{P}_{k}\right)$ and $\mathbf{r}_{\bullet}\left(\mathcal{P}_{\bullet}\right)$. Consider the set

$$
\mathcal{I}=\left\{(x, y) \in X \times X \mid \mathcal{P}_{\bullet}(x) \simeq \mathcal{P}_{\bullet}(y)\right\} .
$$

It is a constructible subset of $X \times X$ and for each $x \in X$ the set

$$
\mathcal{I}(x)=\pi_{1}^{-1}(x) \cap \mathcal{I} \simeq\left\{y \in X \mid \mathcal{P}_{\bullet}(y) \simeq \mathcal{P}_{\bullet}(x)\right\}
$$

is closed in $\mathcal{I}$, hence also constructible. One can easily derive from the standard results on dimensions of fibres [10, Exercise II.3.22] that the set $X_{i}\left(\mathcal{P}_{\bullet}\right)=$ $\{x \in X \mid \operatorname{dim} \mathcal{I}(x) \leq i\}$ is constructible too. (It is also a consequence of Propositions 2.1 and 2.3 below.) We define the number of parameters in the family $\mathcal{P}$. as

$$
\operatorname{par}\left(\mathcal{P}_{\bullet}\right)=\max _{i}\left\{\operatorname{dim} X_{i}\left(\mathcal{P}_{\bullet}\right)-i\right\}
$$

the number of parameters in $\mathbf{I}$-families of vector rank $\mathbf{r}_{\mathbf{0}}$ as

$$
\operatorname{par}\left(\mathbf{r}_{\bullet}, \mathbf{A}, \mathbf{I}\right)=\max \left\{\operatorname{par}\left(\mathcal{P}_{\bullet}\right) \mid \mathbf{r}_{\bullet}\left(\mathcal{P}_{\bullet}\right)=\mathbf{r}_{\bullet}\right\},
$$

and the number of parameters in families of minimal complexes of vector rank $\mathbf{r}$ as

$$
\operatorname{par}\left(\mathbf{r}_{\bullet}, \mathbf{A}\right)=\operatorname{par}\left(\mathbf{r}_{\bullet}, \mathbf{A}, \mathbf{R}\right) .
$$

It is a formal version of the intuitive impression about the number of parameters necessary to define an individual complex inside the family. Of course, we are mainly interested in the "absolute" value $\operatorname{par}(\mathbf{r}, \mathbf{A})$, but further on we need also its "relative" version. Obviously $\operatorname{par}\left(\mathbf{r}_{\bullet}, \mathbf{A}, \mathbf{J}\right) \leq \operatorname{par}\left(\mathbf{r}_{\bullet}, \mathbf{A}, \mathbf{I}\right)$ if $\mathbf{I} \supseteq \mathbf{J}$, especially always $\operatorname{par}\left(\mathbf{r}_{\bullet}, \mathbf{A}, \mathbf{I}\right) \leq \operatorname{par}\left(\mathbf{r}_{\bullet}, \mathbf{A}\right)$.

A family of complexes (2.1) is called non-degenerate if for every point $x \in X$ at least one of the homomorphisms $d_{k}(x)$ is non-zero. Obviously, there is an open set $U \subseteq X$ such that the restriction of $\mathcal{P}$. onto $U$ is non-degenerate, and considering classification problems, as well as calculating parameter numbers, it is enough to deal with non-degenerate families.

We are able, just as in [- $[$ ], to construct some "almost universal" non-degenerate families. It is important that their bases are projective varieties. Namely, fix a vector rank $\mathbf{r}_{\text {• }}$ and set $\mathcal{H}=\mathcal{H}(\mathbf{r}, \mathbf{I})=\bigoplus_{k=m+1}^{n} \mathbf{I}\left(\mathbf{r}_{k}, \mathbf{r}_{k-1}\right)$, where $\mathbf{I}\left(\mathbf{r}, \mathbf{r}^{\prime}\right)=$ $\operatorname{Hom}_{\mathbf{A}}\left(\mathbf{r} A, \mathbf{r}^{\prime} A \mathbf{I}\right)$. Consider the projective space $\mathbb{P}=\mathbb{P}\left(\mathbf{r}_{\bullet}, \mathbf{I}\right)=\mathbb{P}(\mathcal{H})$ and its closed subset $\mathbb{D}=\mathbb{D}\left(\mathbf{r}_{\bullet}, \mathbf{I}\right) \subseteq \mathbb{P}$ consisting of sequences $\left(h_{k}\right)$ such that $h_{k+1} h_{k}=0$ for all $k$. Because of the universal property of projective spaces [10, Theorem II.7.1], the embedding $\mathbb{D}\left(\mathbf{r}_{\bullet}\right) \rightarrow \mathbb{P}\left(\mathbf{r}_{\bullet}\right)$ gives rise to a non-degenerate $\mathbf{I}$-family $\mathcal{V}_{\bullet}=\mathcal{V}_{\bullet}\left(\mathbf{r}_{\bullet}, \mathbf{I}\right)$

$$
\mathcal{V}_{\bullet}\left(\mathbf{r}_{\bullet}\right): \quad \mathcal{V}_{n} \stackrel{d_{n}}{\longrightarrow} \mathcal{V}_{n-1} \stackrel{d_{n-1}}{\longrightarrow} \ldots \longrightarrow \mathcal{V}_{m},
$$

where $\mathcal{V}_{k}=\mathcal{O}_{\mathbb{D}}(n-k) \otimes \mathbf{r}_{k} A$ for all $m \leq k \leq n$. We call $\mathcal{V}_{\bullet}\left(\mathbf{r}_{\bullet}, \mathbf{I}\right)$ the canonical I-family of A-complexes over $\mathbb{D}\left(\mathbf{r}_{\bullet}, \mathbf{I}\right)$. Moreover, morphisms $\phi: X \rightarrow \mathbb{D}\left(\mathbf{r}_{\bullet}, \mathbf{I}\right)$ 
correspond to non-degenerate families of shape (2.1) with $\mathcal{P}_{k}=\mathcal{L}^{\otimes(n-k)} \otimes \mathbf{r}_{k} A$ for some invertible sheaf $\mathcal{L}$ over $X$. Namely, such a family can be obtained as $\phi^{*} \mathcal{V} \bullet$ for a uniquely defined morphism $\phi$.

Proposition 2.1. For every non-degenerate family of $\mathbf{I}$-complexes $\mathcal{P}_{\bullet}$ of vector rank $\mathbf{r}$. over an algebraic variety $X$ there is a finite covering open $X=\bigcup_{j} U_{j}$ such that the restriction of $\mathcal{P}_{\bullet}$ onto each $U_{j}$ is isomorphic to $\phi_{j}^{*} \mathcal{V}\left(\mathbf{r}_{\bullet}, \mathbf{I}\right)$ for some morphism $\phi_{j}: U_{j} \rightarrow \mathbb{D}\left(\mathbf{r}_{\bullet}, \mathbf{I}\right)$.

Proof. For each $x \in X$ there is an open neighbourhood $U \ni x$ such that all restrictions $\mathcal{P}_{k} \mid U$ are isomorphic to $\mathcal{O}_{U} \otimes \mathbf{r}_{k} A$, so the restriction $\mathcal{P}_{\bullet} \mid U$ is obtained from a morphism $U \rightarrow \mathbb{D}\left(\mathbf{r}_{\bullet}, \mathbf{I}\right)$. Evidently it implies the assertion.

Note that morphisms $\phi_{j}$ are not canonical, so we cannot glue them into a global morphism $X \rightarrow \mathbb{D}\left(\mathbf{r}_{\bullet}, \mathbf{I}\right)$.

Corollary 2.2. $\operatorname{par}\left(\mathbf{r}_{\bullet}, \mathbf{A}, \mathbf{I}\right)=\operatorname{par}\left(\mathcal{V}_{\bullet}\left(\mathbf{r}_{\bullet}, \mathbf{I}\right)\right)$.

The main advantage of the families $\mathcal{V}_{\bullet}\left(\mathbf{r}_{\bullet}, \mathbf{I}\right)$ is the following.

Proposition 2.3. All sets

$$
\mathbb{D}_{i}\left(\mathbf{r}_{\bullet}, \mathbf{I}\right)=\left\{x \in \mathbb{D}\left(\mathbf{r}_{\bullet}, \mathbf{I}\right) \mid \operatorname{dim}\left\{y \in \mathbb{D}\left(\mathbf{r}_{\bullet}, \mathbf{I}\right) \mid \mathcal{V}_{\bullet}\left(\mathbf{r}_{\bullet}, \mathbf{I}\right)(y) \simeq \mathcal{V}_{\bullet}\left(\mathbf{r}_{\bullet}, \mathbf{I}\right)(x)\right\} \leq i\right\}
$$

are closed in $\mathbb{D}\left(\mathbf{r}_{\bullet}, \mathbf{I}\right)$.

Proof. Consider the group $G=G\left(\mathbf{r}_{\bullet}\right)=\prod_{k=m}^{n} \operatorname{Aut}_{\mathbf{A}}\left(\mathbf{r}_{k} A\right)$. It acts on $\mathcal{H}\left(\mathbf{r}_{\bullet}, \mathbf{I}\right)$ : $\left(g_{k}\right)\left(h_{k}\right)=\left(g_{k-1} h_{k} g_{k}^{-1}\right)$, hence also on $\mathbb{P}\left(\mathbf{r}_{\bullet}, \mathbf{I}\right)$ and on $\mathbb{D}\left(\mathbf{r}_{\bullet}, \mathbf{I}\right)$. Moreover, complexes $\mathcal{V}\left(\mathbf{r}_{\bullet}, \mathbf{I}\right)(x)$ and $\mathcal{V}\left(\mathbf{r}_{\bullet}, \mathbf{I}\right)(y)$ are isomorphic if and only if the points $x$ and $y$ are in the same orbit of the group $G$. Hence $\mathbb{D}_{i}\left(\mathbf{r}_{\bullet}, \mathbf{I}\right)=\left\{x \in \mathbb{D}\left(\mathbf{r}_{\bullet}, \mathbf{I}\right) \mid \operatorname{dim} G x \leq i\right\}$, and it is well known that this set is closed.

In the next section we shall mainly consider complexes and families of free modules, so we introduce corresponding notations. Let $\mathbf{a}=\left(a_{1}, a_{2}, \ldots, a_{t}\right)=\mathbf{r}(\mathbf{A})$. For any sequence of integers $\mathbf{b}=\left(b_{n}, b_{n-1}, \ldots, b_{m}\right)$ we set $\mathbf{b a}=\left(b_{n} \mathbf{a}, \ldots, b_{m} \mathbf{a}\right)$, $\mathbb{D}(\mathbf{b}, \mathbf{A}, \mathbf{I})=\mathbb{D}(\mathbf{b a}, \mathbf{I})$ and $\operatorname{par}(\mathbf{b}, \mathbf{A}, \mathbf{I})=\operatorname{par}(\mathbf{b a}, \mathbf{A}, \mathbf{I})$, in particular $\operatorname{par}(\mathbf{b}, \mathbf{A})=$ $\operatorname{par}(\mathbf{b a}, \mathbf{A}, \mathbf{R})$. For any $\mathbf{r}=\left(r_{1}, r_{2}, \ldots, r_{t}\right)$ we denote by $] \mathbf{r} / \mathbf{a}[$ the smallest integer $b$ such that $b a_{i} \geq r_{i}$ for all $i$. If $\mathbf{r}_{\bullet}=\left(\mathbf{r}_{n}, \ldots, \mathbf{r}_{m}\right)$, we set $] \mathbf{r} \bullet / \mathbf{a}\left[=(] \mathbf{r}_{n} / \mathbf{a}[, \ldots,] \mathbf{r}_{m} / \mathbf{a}[)\right.$. Just analogous the values $[\mathbf{r} / \mathbf{a}]$ and $\left[\mathbf{r}_{\bullet} / \mathbf{a}\right]$ are defined. If $\left.\mathbf{b}=\right] \mathbf{r}_{\bullet} / \mathbf{a}\left[\right.$ and $\mathbf{b}^{\prime}=\left[\mathbf{r}_{\bullet} / \mathbf{a}\right]$, then evidently

$$
\operatorname{par}\left(\mathbf{b}^{\prime} \mathbf{a}, \mathbf{A}, \mathbf{I}\right) \leq \operatorname{par}\left(\mathbf{r}_{\bullet}, \mathbf{A}, \mathbf{I}\right) \leq \operatorname{par}(\mathbf{b a}, \mathbf{A}, \mathbf{I}) .
$$

Therefore, when we are interested in the asymptotic of the function $\operatorname{par}\left(\mathbf{r}_{\bullet}, \mathbf{a}\right)$ for big ranks, we may only consider complexes of free A-modules.

\section{Families of algebras}

A family of algebras over an algebraic variety $X$ is a sheaf $\mathcal{A}$ of $\mathcal{O}_{X}$-algebras, which is coherent and flat (thus locally free) as a sheaf of $\mathcal{O}_{X}$-modules. For such a family and every sequence $\mathbf{b}=\left(b_{n}, b_{n-1}, \ldots, b_{m}\right)$ one can define the function $\operatorname{par}(\mathbf{b}, \mathcal{A}, x)=\operatorname{par}(\mathbf{b}, \mathcal{A}(x))$. Our main result is the upper semi-continuity of these functions.

Theorem 3.1. Let $\mathcal{A}$ be a family of algebras based on a variety $X$. The set $X_{j}=$ $\{x \in X \mid \operatorname{par}(\mathbf{b}, \mathcal{A}, x) \geq j\}$ is closed for every $\mathbf{b}$ and every integer $j$. 
Proof. We may assume that $X$ is irreducible. Let $\mathbf{K}$ be the field of rational functions on $X$. We consider it as a constant sheaf on $X$. Set $\mathbf{R}=\operatorname{rad}\left(\mathcal{A} \otimes_{\mathcal{O}_{X}} \mathbf{K}\right)$ and $\mathcal{R}=\mathbf{R} \cap \mathcal{A}$. It is a sheaf of nilpotent ideals. Moreover, if $\xi$ is the generic point of $X$, the factor algebra $\mathcal{A}(\xi) / \mathcal{R}(\xi)$ is semisimple. Hence there is an open set $U \subseteq X$ such that $\mathcal{A}(x) / \mathcal{R}(x)$ is semisimple, thus $\mathcal{R}(x)=\operatorname{rad} \mathcal{A}(x)$ for every $x \in U$. Therefore $\operatorname{par}(\mathbf{b}, \mathcal{A}, x)=\operatorname{par}(\mathbf{b}, \mathcal{A}(x), \mathcal{R}(x))$ for $x \in U$, so $X_{j}=X_{j}(\mathcal{R}) \cup X_{j}^{\prime}$, where

$$
X_{j}(\mathcal{R})=\{x \in X \mid \operatorname{par}(\mathbf{b}, \mathcal{A}(x), \mathcal{R}(x)) \geq j\}
$$

and $X^{\prime}=X \backslash U$ is a closed subset in $X$. Using noetherian induction, we may suppose that $X_{j}^{\prime}$ is closed, so we only have to prove that $X_{j}(\mathcal{R})$ is closed too.

Consider the locally free sheaf $\mathcal{H}=\bigoplus_{k=m+1}^{n} \mathcal{H o m}\left(b_{k} \mathcal{A}, b_{k-1} \mathcal{R}\right)$ and the projective space bundle $\mathbb{P}(\mathcal{H})$ [10, Section II.7]. Every point $h \in \mathbb{P}(\mathcal{H})$ defines a set of homomorphisms $h_{k}: b_{k} \mathcal{A}(x) \rightarrow b_{k-1} \mathcal{R}(x)$ (up to a homothety), where $x$ is the image of $h$ in $X$, and the points $h$ such that $h_{k} h_{k+1}=0$ form a closed subset $\mathbb{D} \subseteq \mathbb{P}(\mathcal{H})$. We denote by $\pi$ the restriction onto $\mathbb{D}$ of the projection $\mathbb{P}(\mathcal{H}) \rightarrow X$; it is a projective, hence closed mapping. Moreover, for every point $x \in X$ the fibre $\pi^{-1}(x)$ is isomorphic to $\mathbb{D}(\mathbf{b}, \mathcal{A}(x), \mathcal{R}(x))$. Consider also the group variety $\mathcal{G}$ over $X: \mathcal{G}=\prod_{k=m}^{n} \operatorname{GL}_{b_{k}}(\mathcal{A})$. There is a natural action of $\mathcal{G}$ on $\mathbb{D}$ over $X$, and the sets $\mathbb{D}_{i}=\{z \in \mathbb{D} \mid \operatorname{dim} \mathcal{G} z \leq i\}$ are closed in $\mathbb{D}$. Therefore the sets $Z_{i}=\pi\left(\mathbb{D}_{i}\right)$ are closed in $X$, as well as $Z_{i j}=\left\{x \in Z_{i} \mid \operatorname{dim} \pi^{-1}(x) \geq i+j\right\}$. But $X_{j}(\mathcal{R})=\bigcup_{i} Z_{i j}$, thus it is also a closed set.

\section{Derived tame AND Wild Algebras}

To define derived tame and wild algebras we need consider families of complexes based on non-commutative algebras. As before, we assume that the field $\mathbb{k}$ is algebraically closed, though the definitions do not use this restriction.

Definition 4.1. Let $\mathbf{A}$ be a finite dimensional algebra over the field $\mathbb{k}$ with radical $\mathbf{R}$ and $\mathbf{B}$ be any $\mathbb{k}$-algebra.

1. A family of minimal $\mathbf{A}$-complexes based on $\mathbf{B}$ is defined as a complex

$$
\mathcal{P}_{\bullet}: \quad \mathcal{P}_{n} \stackrel{d_{n}}{\longrightarrow} \mathcal{P}_{n-1} \stackrel{d_{n-1}}{\longrightarrow} \ldots \longrightarrow \mathcal{P}_{m},
$$

of finitely generated projective $\mathbf{B}^{\circ} \otimes \mathbf{A}$-modules such that $\operatorname{Im} d_{k} \subseteq \mathcal{P}_{k-1} \mathbf{R}$ for all $k$.

2. For a family (4.1) and a finite dimensional (over $\mathbb{k}$ ) left $\mathbf{B}$-module $L$ we denote by $\mathcal{P}_{\bullet}(L)$ the complex

$$
L \otimes_{\mathcal{B}} \mathcal{P}_{n} \stackrel{1 \otimes d_{n}}{\longrightarrow} L \otimes_{\mathbf{B}} \mathcal{P}_{n-1} \stackrel{1 \otimes d_{n-1}}{\longrightarrow} \ldots \longrightarrow L \otimes_{\mathbf{B}} \mathcal{P}_{m} .
$$

3. We call a family (4.1) strict if

(a) $\mathcal{P}_{\bullet}(L) \simeq \mathcal{P}_{\bullet}\left(L^{\prime}\right)$ if and only if $L \simeq L^{\prime}$;

(b) $\mathcal{P}(L)$ is indecomposable if and only if $L$ is indecomposable.

4. We call $\mathbf{A}$ derived wild if for every finitely generated $\mathbb{k}$-algebra $\mathbf{B}$ there is a strict family of minimal A-complexes based on $\mathbf{B}$.

5. We call $\mathbf{A}$ derived tame if there is a set $\mathfrak{M}$ of families of minimal $\mathbf{A}$-complexes with the following properties:

(a) Every $\mathcal{P}_{\bullet} \in \mathfrak{M}$ is based on a rational algebra $\mathbf{B}$, which means that $\mathbf{B} \simeq \mathbb{k}\left[x, f(x)^{-1}\right]$ for a non-zero polynomial $f(x)$. We define $\mathbf{r}_{\bullet}\left(\mathcal{P}_{\bullet}\right)$ as $\mathbf{r}_{\bullet}\left(\mathcal{P}_{\bullet}(L)\right)$ for some (hence any) one-dimensional B-module $L$. 
(b) The set

$$
\mathfrak{M}\left(\mathbf{r}_{\bullet}\right)=\left\{\mathcal{P}_{\bullet} \in \mathfrak{M} \mid \mathbf{r}_{\bullet}\left(\mathcal{P}_{\bullet}\right)=\mathbf{r}_{\bullet}\right\}
$$

is finite for each $\mathbf{r}_{\bullet}$.

(c) For every $\mathbf{r}_{\bullet}$ all indecomposable minimal $\mathbf{A}$-complexes of vector rank $\mathbf{r}_{\bullet}$, except maybe finitely many isomorphism classes of such complexes, are isomorphic to $\mathcal{P}_{\bullet}(L)$ for some $\mathcal{P}_{\bullet} \in \mathfrak{M}$ and some $\mathbf{B}$-module $L$.

Remark. These definitions do not coincide but are easily seen to be equivalent to other used definitions of derived tame and wild algebras, for instance those of 8, 2, 9. As usually, to show that $\mathbf{A}$ is derived wild it suffices to construct a strict family over one of specimen algebras such as free algebra $\mathbb{k}\langle x, y\rangle$, or polynomial algebra $\mathbb{k}[x, y]$, or power series algebra $\mathbb{k}[[x, y]]$.

The following proposition follows from elementary geometrical consideration like in 3 .

Proposition 4.2. $\quad$ 1. If $\mathbf{A}$ is derived tame, then $\operatorname{par}(\mathbf{b}, \mathbf{A}) \leq|\mathbf{b}| \operatorname{dim} \mathbf{A}$ for each sequence $\mathbf{b}=\left(b_{n}, b_{n-1}, \ldots, b_{m}\right)$, where $|\mathbf{b}|=\sum_{k=m}^{n} b_{k}$.

2. If $\mathbf{A}$ is derived wild, then there is a sequence $\mathbf{b}$ such that $\operatorname{par}(c \mathbf{b}, \mathbf{A}) \geq c^{2}$ for every integer $c$.

In particular, no algebra can simultaneously be both derived tame and derived wild.

In what follows we use the following supposition, which is believed by most experts.

Supposition 4.3. Every finite dimensional algebra is either derived tame or derived wild.1

Corollary 4.4. Let $\mathcal{A}$ be a family of algebras based on a algebraic variety $X$. Then $W=\{x \in X \mid \mathcal{A}(x)$ is derived wild $\}$ is a union of a countable sequence of closed subsets.

Proof. Indeed $W=\bigcup_{c, \mathbf{b}} W_{c, \mathbf{b}}$, where $\left.W_{c, \mathbf{b}}=\{x \in X|\operatorname{par}(c \mathbf{b}, \mathcal{A}(x))>c| \mathbf{b} \mid \operatorname{rk} \mathcal{A})\right\}$. By Theorem 3.1 all these subsets are closed in $X$.

Conjecture 4.5. In the situation of Corollary 4.4, the set $W$ is always closed in $X$, or, the same, the set $\{x \in X \mid \mathcal{A}(x)$ is tame $\}$ is open.

Corollary 4.6. Suppose that an algebra A, which is derived wild, degenerates to another algebra $\overline{\mathbf{A}}$, i.e. there is a family of algebras $\mathcal{A}$ based on a variety $X$ such that $\mathcal{A}(x) \simeq \mathbf{A}$ for all $x$ from a dense open subset $U_{X}$ and there is a point $y \in X$ such that $\mathcal{A}(y) \simeq \overline{\mathbf{A}}$. Then $\overline{\mathbf{A}}$ is also derived wild.

Remark 4.7. Recently Th. Brüstle has announced a counter-example to the semicontinuity for derived categories (cf. [2, Section 8.1]). Namely, he claims that the

\footnotetext{
${ }^{1}$ Now V. Bekkert and the author are preparing an article with a proof of this conjecture.
} 
derived wild algebra $\mathbf{A}$ given by the quiver with relations

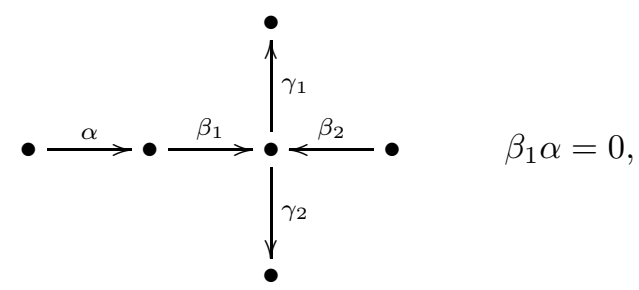

degenerates to the derived tame algebra $\overline{\mathbf{A}}$ given by the quiver with relations

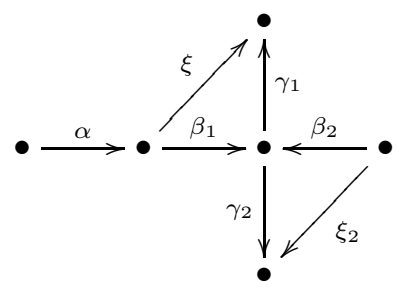

$$
\beta_{1} \alpha=\gamma_{1} \beta_{1}=\gamma_{2} \beta_{2}=0
$$

As a matter of fact, it is not an example, since $\operatorname{dim} \mathbf{A}=15$, while $\operatorname{dim} \overline{\mathbf{A}}=16$, so the latter cannot be a degeneration of the former.

\section{REFERENCES}

[1] Bass, H. Finitistic dimension and a homological generalization of semi-primary rings. Trans. Amer. Math. Soc. 95 (1960), 466-488.

[2] Brüstle, Th. Tree Algebras and Quadratic Forms. Habilitation Thesis. Universität Bielefeld, 2002.

[3] Drozd, Yu. On tame and wild matrix problems. In: Matrix Problems, Institute of Mathematics, Kiev, 1977, 104-114.

[4] Drozd, Yu. Reduction algorithm and representations of boxes and algebras. Comptes Rendues Math. Acad. Sci. Canada 23 (2001), 97-125.

[5] Drozd, Yu. and Greuel, G.-M. Semi-continuity for Cohen-Macaulay modules, Math. Ann. 306 (1996), 371-389.

[6] Gabriel, P, Roiter, A. Representation of Finite-Dimensional Algebras. Encyclopaedia of Mathematical Sciences, vol. 73 (Algebra VIII). Springer-Verlag, Berlin-HeidelbergNew York, 1992.

[7] Geiss, Ch. On degenerations of tame and wild algebras. Arch. Math. 64 (1995), 11-16.

[8] Geiß, Ch. Derived tame algebras and Euler-forms. Math. Z. 239 (2002), 829-862.

[9] Geiß, Ch. and Krause, H. On the notion of derived tameness. Preprint, 2000.

[10] Hartshorn, R. Algebraic Geometry. Springer-Verlag, New York - Heidelberg - Berlin, 1997.

[11] Huisgen-Zimmermann, B. and Saorín, M. Geometry of chain complexes and outer automorphisms under derived equivalence. Trans. Amer. Math. Soc. 353 (2001), 4757-4777.

[12] Knörrer, H. Torsionsfreie Moduln bei Deformation von Kurvensingularitäten. Singularities, Representation of Algebras and Vector Bundles, Lambrecht 1985 (Eds.: Greuel, G.-M.; Trautmann, G.). Lecture Notes in Math., Vol. 1273, pp. 150-155. Berlin-Heidelberg-New York: Springer 1987.

Kyiv Taras Shevchenko University, Department of Mechanics and Mathematics, 01033 KYIV, UKRAINE

E-mail address: yuriy@drozd.org 